\title{
Metais pesados em girassol adubado com lodo de esgoto submetido a diferentes processos de estabilização
}

\author{
Altina L. Nascimento ${ }^{1}$, Regynaldo A. Sampaio ${ }^{2}$, Suely F.da Cruz ${ }^{3}$, \\ Geraldo R. Zuba Junio ${ }^{4}$, Cristiane F. Barbosa ${ }^{5}$ \& Luiz A. Fernandes ${ }^{6}$ \\ ${ }^{1}$ ESALQ/USP. Piracicaba, SP. E-mail: altinalacerda@yahoo.com.br (Autora correspondente) \\ ${ }^{2}$ ICA/UFMG. Montes Claros, MG. E-mail: rsampaio@ufmg.br \\ ${ }^{3}$ ICA/UFMG. Montes Claros, MG. E-mail: suelysema@yahoo.com.br \\ ${ }^{4}$ ICA/UFMG. Montes Claros, MG. E-mail: juniozuba@yahoo.com.br \\ ${ }^{5}$ ICA/UFMG. Montes Claros, MG. E-mail: crisfbagro@hotmail.com \\ ${ }^{6}$ ICA/UFMG. Montes Claros, MG. E-mail: larnaldo@ufmg.br
}

\section{Palavras-chave:}

Helianthus annuus

biossólido

compostagem

vermicompostagem

lodo caleado

\begin{abstract}
R E S U M O
O lodo de esgoto apresenta grande potencial para utilização agrícola; entretanto, esta forma de disposição final causa preocupação uma vez que existe a possibilidade de inserção de metais pesados na cadeia alimentar. Objetivou-se, com este trabalho, avaliar os teores de metais pesados no solo e na planta de girassol em resposta à adubação com lodo de esgoto submetido a diferentes processos de estabilização. O experimento foi desenvolvido em Cambissolo Háplico com os tratamentos: testemunha (sem adubação), adubação com lodo de esgoto solarizado, adubação com lodo de esgoto compostado, adubação com lodo de esgoto vermicompostado, adubação com lodo de esgoto caleado e adubação química recomendada para a cultura. Utilizou-se o delineamento experimental em blocos casualizados com 4 repetições. Independente da forma de estabilização, a aplicação de lodo de esgoto não influenciou os teores de $\mathrm{Cu}, \mathrm{Cr}$, Cd e Ni no solo porém aumentou os teores de Zn. A aplicação de lodo de esgoto solarizado e de lodo de esgoto caleado aumentou os teores de $\mathrm{Pb}$ no solo; de modo geral, os teores de metais pesados na folha e no pecíolo do girassol não foram influenciados pela aplicação de lodo de esgoto.
\end{abstract}

Key words: Helianthus annuus biosolids composting vermicomposting limed sludge

\section{Heavy metals in sunflower fertilized with sewage sludge under different stabilization processes}

\begin{abstract}
A B S T R A C T
Sewage sludge has a great potential for agricultural use, however, this form of disposal is of concern since there is possibility of heavy metals insertion in food chain. The objective of this study was to evaluate the contents of heavy metals in soil and sunflower plant in response to fertilization with sewage sludge under different stabilization processes. The experiment was conducted in Haplic Cambisol, with the treatments: control (without fertilization), fertilization with solarized sewage sludge, fertilization with composted sewage sludge, fertilization with vermicomposted sewage sludge, fertilization with limed sewage sludge and chemical fertilizer recommended for the crop. A randomized block design with four repetitions was used. Independent of stabilization method, the application of sewage sludge did not affect the $\mathrm{Cu}, \mathrm{Cr}, \mathrm{Cd}$ and $\mathrm{Ni}$ concentrations in the soil, but increased $\mathrm{Zn}$ levels. Also, the application of solarized sewage sludge and of the limed sewage sludge increased $\mathrm{Pb}$ content in soil. In general the contents of heavy metals in sunflower leaf and petiole were not influenced by sewage sludge application.
\end{abstract}

\section{INTRODUÇÃo}

O lodo de esgoto, resíduo sólido do processo de tratamento de esgotos, contém matéria orgânica e nutrientes que exercem importante papel na produção agrícola e na manutenção da fertilidade do solo; além disso, a matéria orgânica contida neste resíduo aumenta o conteúdo de húmus reduzindo a densidade do solo, melhorando a capacidade de armazenamento e infiltração de água, aumentando a estabilidade dos agregados e reduzindo a erosão (Galdos et al., 2004; Souza et al., 2005; Corrêa et al., 2009; Maria et al., 2010; Campos et al., 2011).
Por outro lado, a possibilidade de contaminação do solo é motivo de preocupação quando este resíduo é usado na agricultura, sendo que os principais contaminantes presentes em lodo de esgoto são metais pesados, tais como chumbo $(\mathrm{Pb})$, níquel $(\mathrm{Ni})$, cádmio $(\mathrm{Cd})$, cromo $(\mathrm{Cr})$, cobre $(\mathrm{Cu})$ e zinco (Zn), organismos patogênicos (bactérias, protozoários, vírus etc.), além de uma gama de compostos orgânicos sintéticos (hidrocarbonetos policíclicos aromáticos, dioxinas, furanos, pesticidas, hormônios sintéticos e naturais etc).

Quando o lodo é aplicado ao solo os contaminantes podem ocasionar toxidez para as plantas e microrganismos do solo 
podem lixiviar-se, acumular-se nas plantas e, por vezes, serem transferidos para animais; por outro lado, existe a possibilidade de animais ingerirem solo contaminado com consequente inserção de tais contaminantes na cadeia alimentar (During \& Gath, 2002; Haynes et al., 2009). Assim sendo, para utilização agrícola do lodo de esgoto faz-se necessária sua estabilização e diversos métodos são utilizados como, por exemplo, solarização, compostagem, vermicompostagem e caleação.

Durante o processo de compostagem e vermicompostagem as moléculas orgânicas são quebradas e os elementos químicos presentes nessas moléculas passam para formas mais fitodisponíveis. Khwairakpam \& Bhargava (2009) observaram que o processo de vermicompostagem reduziu os níveis dos metais $\mathrm{Mn}, \mathrm{Zn}, \mathrm{Pb}$ e $\mathrm{Cu}$ em lodo de esgoto. Como não houve lixiviação de metais pela drenagem de água este resultado foi atribuído ao acúmulo de metais pesados nas minhocas fato também observado por Morgan \& Morgan (1998) e Vijver et al. (2007). Resultado diferente foi constatado por Gupta \& Garg (2008) os quais observaram que o processo de vermicompostagem aumentou os teores de metais pesados no lodo de esgoto. De acordo com Azizi et al. (2013) o aumento ou redução nos teores de metais pesados em vermicomposto de lodo de esgoto apresenta relação com o tempo de estabilização sendo que após a décima quinta semana ocorre excreção de metais pesados pelas minhocas, com consequente aumento da sua concentração no vermicomposto.

O processo de estabilização química do lodo de esgoto consiste na adição de produtos para inibir a atividade biológica ou oxidar a matéria orgânica sendo a cal um dos produtos químicos mais utilizados em razão do baixo custo, simplicidade do processo e eficiência na eliminação de patógenos. A eliminação de patógenos do lodo de esgoto pelo processo de caleação é resultado da elevação da temperatura e do $\mathrm{pH}$ do resíduo, que chega a valores próximos de 12 e, como consequência da elevação do $\mathrm{pH}$, ocorre a insolubilização de metais (Fia et al., 2005).

A utilização agrícola do lodo de esgoto tem sido bastante estudada. Entretanto, são poucos os trabalhos que relatam a associação desse resíduo com plantas de girassol, espécie considerada acumuladora de metais sendo utilizada em diversos trabalhos de fitorremediação (Cutright et al., 2010; Fassler et al., 2010). Além disto, considerando que as formas de estabilização do lodo de esgoto alteram a disponibilidade de metais para as culturas, os resultados obtidos com a utilização agrícola desse resíduo podem variar consideravelmente; assim, este trabalho teve como objetivo avaliar os teores de metais pesados no solo e em plantas de girassol adubadas com lodo de esgoto submetido a diferentes processos de estabilização.

\section{Material e Métodos}

O experimento foi realizado no período de julho a novembro de 2010, no Instituto de Ciências Agrárias da Universidade Federal de Minas Gerais, localizado em Montes Claros - MG, latitude $16^{\circ} 51^{\prime} 38^{\prime \prime}$ 'S e longitude $44^{\circ} 55^{\prime} 00^{\prime \prime}$ W, em Cambissolo Háplico, cujas características químicas e físicas são apresentadas na Tabela 1.
Tabela 1. Características químicas e físicas do solo da área experimental ${ }^{1}$

\begin{tabular}{|c|c|c|}
\hline \multirow{2}{*}{ Atributos do solo } & \multicolumn{2}{|c|}{ Camada (cm) } \\
\hline & $0-20$ & $20-40$ \\
\hline pH em água & 5,80 & 5,50 \\
\hline P Mehlich $\left(\mathrm{mg} \mathrm{kg}^{-1}\right)$ & 3,50 & 1,90 \\
\hline$P$ remanescente $\left(\mathrm{mg} \mathrm{L}^{-1}\right)$ & 16,70 & 14,40 \\
\hline $\mathrm{K}\left(\mathrm{mg} \mathrm{dm}^{-3}\right)$ & 229,00 & 117,00 \\
\hline $\mathrm{Ca}\left(\mathrm{cmol}_{\mathrm{c}} \mathrm{dm}^{-3}\right)$ & 3,60 & 2,80 \\
\hline $\mathrm{Mg}\left(\mathrm{cmol}_{\mathrm{c}} \mathrm{dm}^{-3}\right)$ & 1,50 & 0,80 \\
\hline $\mathrm{Al}\left(\mathrm{cmol}_{\mathrm{c}} \mathrm{dm}^{-3}\right)^{\prime}$ & 0,20 & 0,76 \\
\hline $\mathrm{Zn}\left(\mathrm{mg} \mathrm{dm}^{-3}\right)$ & 0,80 & 0,30 \\
\hline $\mathrm{Cu}\left(\mathrm{mg} \mathrm{dm} \mathrm{d}^{-3}\right)$ & 0,80 & 0,30 \\
\hline $\mathrm{Pb}\left(\mathrm{mg} \mathrm{dm}^{-3}\right)$ & 10,10 & 10,90 \\
\hline $\mathrm{Ni}\left(\mathrm{mg} \mathrm{dm}^{-3}\right)$ & 0,00 & 0,00 \\
\hline $\mathrm{Cd}\left(\mathrm{mg} \mathrm{dm}^{-3}\right)$ & 0,00 & 0,00 \\
\hline $\mathrm{Cr}\left(\mathrm{mg} \mathrm{dm}^{-3}\right)$ & 0,00 & 0,00 \\
\hline $\mathrm{H}+\mathrm{Al}\left(\mathrm{cmol}_{\mathrm{c}} \mathrm{dm}^{-3}\right)$ & 3,62 & 3,62 \\
\hline Soma de Bases $\left(\mathrm{cmol}_{\mathrm{c}} \mathrm{dm}^{-3}\right)$ & 5,69 & 3,90 \\
\hline CTC efetiva $\left(\mathrm{cmol}_{\mathrm{C}} \mathrm{dm}^{-3}\right)$ & 5,89 & 4,66 \\
\hline Saturação por alumínio (\%) & 3,00 & 16,00 \\
\hline CTC potencial $\left(\mathrm{cmol}_{\mathrm{c}} \mathrm{dm}^{-3}\right)$ & 9,31 & 7,52 \\
\hline Saturação por bases (\%) & 61,00 & 52,00 \\
\hline Matéria orgânica $\left(\right.$ dag $\left.\mathrm{kg}^{-1}\right)$ & 3,39 & 2,50 \\
\hline Areia grossa $\left(\mathrm{dag} \mathrm{kg}^{-1}\right)$ & 5,60 & 4,30 \\
\hline Areia fina $\left(\right.$ dag $\left.^{-1}\right)$ & 14,40 & 21,70 \\
\hline Silte $\left(\right.$ dag $\left.\mathrm{kg}^{-1}\right)$ & 38,00 & 36,00 \\
\hline $\operatorname{Argila}\left(\right.$ dag $\left.\mathrm{kg}^{-1}\right)$ & 42,00 & 38,00 \\
\hline
\end{tabular}

'EMBRAPA (1997)

Utilizou-se o delineamento estatístico de blocos casualizados com 6 tratamentos e 4 repetições, ou seja, 1 tratamento químico $\left(20 \mathrm{~kg} \mathrm{ha}^{-1}\right.$ de N, $70 \mathrm{~kg} \mathrm{ha}^{-1}$ de $\mathrm{P}_{2} \mathrm{O}_{5}$ e $30 \mathrm{~kg} \mathrm{ha}^{-1}$ de $\mathrm{K}_{2} \mathrm{O}$, no plantio e $40 \mathrm{~kg} \mathrm{ha}^{-1}$ de N, 45 dias após emergência) de acordo com as Recomendações para o uso de corretivos e fertilizantes em Minas Gerais (CFSEMG, 1999) utilizando-se, como fontes de $\mathrm{N}, \mathrm{P}$ e K respectivamente, ureia, superfosfato simples e cloreto de potássio, 4 tratamentos com lodo de esgoto estabilizado por diferentes formas aplicados com base nos teores de nitrogênio disponível, calculados de acordo com metodologias e fórmulas descritas na Resolução CONAMA 375, de agosto de 2006 (Brasil, 2006) assim definidos: solarizado $\left(6,12 \mathrm{t} \mathrm{ha}^{-1}\right)$, compostado $\left(37,27 \mathrm{t} \mathrm{ha}^{-1}\right)$, vermicompostado $\left(19,17 \mathrm{tha}^{-1}\right)$ e caleado $(50,42$ $\mathrm{t} \mathrm{ha}^{-1}$ ) e um tratamento sem adubação. Como planta indicadora utilizou-se o híbrido simples de girassol Helio 863.

O lodo de esgoto solarizado foi coletado na estação de tratamento de esgoto (ETE) do município de Juramento, MG. A ETE possui linha de tratamento composta de tratamento preliminar e de reator anaeróbio UASB interligado a uma lagoa de pós-tratamento do tipo facultativa e tratamento do lodo de esgoto por meio do processo de solarização em leito de secagem. Para a compostagem misturou-se o lodo de esgoto solarizado à poda de grama cuja espécie predominante era Paspalum notatum (grama-batatais), de forma a se obter uma relação C:N de 30:1. Constantemente, foram monitoradas a temperatura e a umidade e, para controle desses fatores foram efetuados revolvimentos sistemáticos das pilhas de composto.

Com vista à obtenção do vermicomposto fez-se um précomposto de lodo de esgoto misturado à poda de grama, utilizado após um mês do início do processo de decomposição como substrato para a vermicompostagem com minhocas 
vermelhas da Califórnia (Eisenia foetida). O lodo de esgoto caleado foi obtido pela adição de cal virgem em quantidade correspondente a 50\% da massa seca de lodo de esgoto; após a mistura elevou-se a umidade dos materiais a 70\%. Os teores de metais nos materiais contendo lodo de esgoto e as quantidades aplicadas em cada tratamento são apresentados na Tabela 2.

As adubações referentes aos tratamentos com lodo de esgoto foram realizadas de uma única vez, no sulco de plantio. As parcelas experimentais se constituíram de quatro linhas de 3,60 $\mathrm{m}$ de comprimentos, espaçadas $0,80 \mathrm{~m}$, sendo a parcela útil as duas linhas centrais desprezando-se $0,4 \mathrm{~m}$ de cada extremidade. O semeio foi realizado em sulcos colocando-se três sementes em cada local de semeio a uma distância de 0,20 m entre plantas. Quinze dias após a emergência fez-se o desbaste deixando-se apenas uma planta por cova. Manteve-se a área de cultivo sem a presença de plantas daninhas por meio de capina manual e se fez o fornecimento de água à cultura, por meio de irrigação por aspersão.

No início do florescimento do girassol foram coletadas amostras de folhas e pecíolos do terço superior de 10 plantas escolhidas aleatoriamente na parcela útil, para análise química dos teores de $\mathrm{Zn}, \mathrm{Cu}, \mathrm{Pb}, \mathrm{Cr}, \mathrm{Cd}$ e Ni (Tedesco et al., 1995; Malavolta et al., 1997).

Quando o girassol se apresentava na fase (R9) cujos capítulos se encontravam voltados para baixo, realizou-se a colheita, após a qual se coletou, entre plantas na linha, nas profundidades de 0-20 e 20-40 cm, 8 subamostras de solo por parcela para formar amostras compostas para análises de $\mathrm{Zn}, \mathrm{Cu}, \mathrm{Pb}, \mathrm{Cr}, \mathrm{Cd}, \mathrm{Ni}$, (Tedesco et al., 1995).

Os dados obtidos foram submetidos à análise de variância e as médias dos tratamentos comparadas até $5 \%$ de probabilidade pelo teste Scott-Knott.

\section{Resultados e Discussão}

Apesar de presentes no lodo de esgoto e nos adubos químicos (Tabela 2) os teores de Cd e Ni no solo estiveram abaixo dos níveis de detecção pelo método analítico utilizado; além disto, para nenhum dos metais observados no solo os teores estiveram acima do considerado seguro de acordo com a United States Environmental Protection Agency (USEPA, 1999) e as quantidades aplicadas não ultrapassaram a carga acumulada teórica permitida pela resolução CONAMA no 375 , de agosto de 2006 (Brasil, 2006).

A aplicação de lodo de esgoto influenciou os teores de $\mathrm{Zn}$ no solo, na camada de $0-20 \mathrm{~cm}$, apresentando maiores valores que os observados nos tratamentos testemunha e químico, independentemente da forma de estabilização do lodo (Tabela 3). Na profundidade de $0-20 \mathrm{~cm}$ no tratamento testemunha (sem adubação) e no tratamento químico, os teores de $\mathrm{Zn}$

Tabela 2. Metais pesados ${ }^{1}$ no lodo de esgoto solarizado, compostado, vermicompostado e caleado e nos adubos químicos utilizados no experimento

\begin{tabular}{|c|c|c|c|c|c|c|}
\hline \multirow{2}{*}{ Lodo de esgoto } & $\mathrm{Zn}$ & $\mathrm{Cu}$ & $\mathrm{Ni}$ & Cd & $\mathrm{Pb}$ & $\mathrm{Cr}$ \\
\hline & \multicolumn{6}{|c|}{$\mathrm{mg} \mathrm{kg}^{-1}$} \\
\hline Compostado & $(15,02)$ & $(2,35)$ & $(1,73)$ & $(0,05)$ & $(4,36)$ & $(33,54)$ \\
\hline \multirow{2}{*}{ Vermicompostado } & 374 & 71 & 43,00 & 1,21 & 119,51 & 850 \\
\hline & $(7,17)$ & $(1,36)$ & $(0,82)$ & $(0,02)$ & $(2,29)$ & $(16,29)$ \\
\hline Superfosfato simples & 142 & 16,2 & 62 & 6,2 & 116 & 333 \\
\hline Ureia & 2,5 & 1,62 & - & 0,7 & 8 & - \\
\hline Cloreto de potássio & 2,5 & 4,62 & 15 & 2,4 & 83 & - \\
\hline CMP & 2.800 & 1.500 & 420 & 39 & 300 & 1.000 \\
\hline
\end{tabular}

${ }^{1}$ Tedesco et al. (1995); CMP - concentração máxima permitida no lodo de esgoto ou produto derivado (Brasil, 2006); os valores entre parênteses correspondem às quantidades de metais aplicadas ao solo em kg ha-1

Tabela 3. Teores de metais no solo em resposta à aplicação de fertilizante mineral e lodo de esgoto tratado de diferentes formas

\begin{tabular}{|c|c|c|c|c|c|c|c|c|c|}
\hline \multirow[b]{2}{*}{ Metal } & \multirow{2}{*}{$\begin{array}{l}\text { Profundidade } \\
\text { cm }\end{array}$} & \multicolumn{6}{|c|}{ Tratamentos } & \multirow{2}{*}{$\begin{array}{c}\text { CV } \\
\%\end{array}$} & \multirow{2}{*}{$\begin{array}{l}\text { Limite }^{1} \\
\mathrm{mg} \mathrm{dm}^{-3}\end{array}$} \\
\hline & & TE & LS & LCO & LV & LCA & $\mathbf{A Q}$ & & \\
\hline $\mathrm{Zn}$ & $0-20$ & $0,83 \mathrm{~b}$ & $2,40 \mathrm{a}$ & $3,77 \mathrm{a}$ & $3,50 \mathrm{a}$ & $4,02 \mathrm{a}$ & $0,78 \mathrm{~b}$ & 43,79 & 2.800 \\
\hline \multirow[b]{2}{*}{$\mathrm{Cu}$} & $0-20$ & $0,47^{\mathrm{ns}}$ & $0,60^{\mathrm{ns}}$ & $0,55^{\mathrm{ns}}$ & $0,55^{\mathrm{ns}}$ & $0,55^{\mathrm{ns}}$ & $0,45^{\mathrm{ns}}$ & 20,29 & \multirow{2}{*}{1.500} \\
\hline & $20-40$ & $0,33^{\text {ns }}$ & $0,33^{\text {ns }}$ & $0,35^{\text {ns }}$ & $0,38^{\text {ns }}$ & $0,32^{\text {ns }}$ & $0,35^{\mathrm{ns}}$ & 20,23 & \\
\hline $\mathrm{Pb}$ & $0-20$ & $9,00 \mathrm{~b}$ & $11,23 \mathrm{a}$ & $8,08 \mathrm{~b}$ & $7,33 \mathrm{~b}$ & $12,68 \mathrm{a}$ & $9,73 \mathrm{~b}$ & 18,53 & 300 \\
\hline $\mathrm{Cr}$ & $20-40$ & $4,67^{\mathrm{ns}}$ & $3,28^{\mathrm{ns}}$ & $6,55^{\mathrm{ns}}$ & $4,22^{\mathrm{ns}}$ & $9,30^{\mathrm{ns}}$ & $9,33^{\mathrm{ns}}$ & 66,79 & - \\
\hline
\end{tabular}

TE - Testemunha; LS - Lodo de esgoto solarizado; LCO - Lodo de esgoto compostado; LV - Lodo de esgoto vermicompostado; LCA - Lodo de esgoto caleado; AQ - Adubação Química; ns - Não significativo ${ }^{1}$ Adaptado de USEPA (1999), considerando a densidade do solo igual a $1 \mathrm{~g} \mathrm{~cm}^{-3}$ e $20 \mathrm{~cm}$ de profundidade

Para cada nutriente, médias seguidas de mesma letra na linha não diferem estatisticamente entre si a 0,05 de probabilidade pelo teste Scott-Knott 
foram classificados, de acordo com Galrão (2004) baixos (0-1,0 $\mathrm{mg} \mathrm{dm}^{-3}$ ) e nos tratamentos com lodo de esgoto altos $(>1,6$

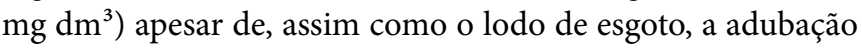
química, em especial a fosfatada, ser fonte do elemento, como se observa na Tabela 2.

Considerando as quantidades de lodo de esgoto aplicadas e os teores de $\mathrm{Zn}$ no lodo, foram adicionadas ao solo, por meio do lodo de esgoto solarizado, compostado, vermicompostado e caleado, quantidades respectivas de 3,22; 15,02; 7,17 e 22,89 $\mathrm{kg} \mathrm{ha}^{-1}$ de Zn enquanto segundo Galrão (2004) em solos com baixo teor de $\mathrm{Zn}$, como o do presente experimento, recomendase a aplicação de $6 \mathrm{~kg} \mathrm{ha}^{-1}$ do micronutriente para o cultivo de girassol. Não foram observadas diferenças estatísticas para os teores de $\mathrm{Zn}$, na profundidade de $20-40 \mathrm{~cm}$ apesar de, quando aplicado lodo de esgoto caleado, os teores do micronutriente nessa camada do solo terem sido classificados médios e nos demais tratamentos, baixos (Galrão, 2004).

O lodo de esgoto utilizado no presente experimento teve origem basicamente doméstica, sendo que esse tipo de lodo de esgoto geralmente é rico em $\mathrm{Zn}$, de vez que este elemento está presente em diversos produtos de uso domestico como, por exemplo, cremes para a pele, pomadas, maquiagem, desodorante, talco, shampoo, creme de barbear etc (Haynes et al., 2009; Houhou et al., 2009). O aumento de $\mathrm{Zn}$ em solo adubado com lodo de esgoto também foi observado por Borges \& Coutinho (2004b), Zuba Junio et al. (2011) e Nogueira et al. (2013).

A aplicação de lodo de esgoto não influenciou os teores de $\mathrm{Cu}$ no solo, na profundidade de $0-40 \mathrm{~cm}$ (Tabela 3 ), embora tenham sido aplicadas via lodo de esgoto solarizado, compostado, vermicompostado e caleado, quantidades respectivas de 0,49 , 2,35, 1,36 e 3,58 $\mathrm{kg} \mathrm{ha}^{-1} \mathrm{de} \mathrm{Cu}$. Considerando o nível médio de $\mathrm{Cu}$ no solo antes da instalação do experimento, Galrão (2004) recomenda a aplicação de $0,5 \mathrm{~kg} \mathrm{ha}^{-1}$ de $\mathrm{Cu}$ para o cultivo do girassol. Contudo, apesar de nos tratamentos com lodo de esgoto compostado, vermicompostado e caleado, a quantidade aplicada ter sido superior à recomendada, não foram constatados sintomas de toxidez na cultura.
A adição de $\mathrm{Pb}$ ao solo com a aplicação de lodo de esgoto solarizado, compostado, vermicompostado e caledo foi de 0,$57 ; 4,36 ; 2,29$ e $14,39 \mathrm{~kg} \mathrm{ha}^{-1}$, respectivamente. Ressaltase que esses valores estão bem abaixo da carga acumulada teórica permitida pela resolução Conama 375 (Brasil, 2006) que é de $41 \mathrm{~kg} \mathrm{ha}^{-1}$ de $\mathrm{Pb}$. Observa-se, na Tabela 3, que os teores de $\mathrm{Pb}$ no solo, na camada de $0-20 \mathrm{~cm}$, quando aplicado lodo de esgoto solarizado ou caleado, foram superiores aos observados nos tratamentos com lodo de esgoto compostado, vermicompostado, adubação química e não adubação do solo (testemunha). Na profundidade de $20-40 \mathrm{~cm}$ não foram constatadas diferenças estatísticas para os teores de $\mathrm{Pb}$ no solo. Contrário ao observado neste experimento, Nogueira et al. (2007) observaram reduções nos teores de $\mathrm{Pb}$ no solo, quando aplicado lodo de esgoto caleado comparado com as outras formas de estabilização e atribuíram tal fato à redução da disponibilidade do elemento pelo aumento do $\mathrm{pH}$ do solo. A diferença observada nos resultados desses experimentos pode estar relacionada à quantidade de $\mathrm{Pb}$ aplicada ao solo uma vez que o aporte de $\mathrm{Pb}$ ao solo por esses autores com a aplicação de lodo de esgoto caleado foi de apenas 0,92 $\mathrm{kg} \mathrm{ha}^{-1}$ enquanto no presente experimento houve um aporte de $14,38 \mathrm{~kg} \mathrm{ha}^{-1}$.

Foram adicionadas ao solo quantidades respectivas de 4,29; 33,$54 ; 16,29$ e 41,60 $\mathrm{kg} \mathrm{ha}^{-1} \mathrm{de} \mathrm{Cr}$, via lodo de esgoto solarizado, compostado, vermicompostado e caleado, além de o metal se encontrar presente também no adubo fosfatado (Tabela 2). Entretanto, os teores de $\mathrm{Cr}$ no solo foram estatisticamente iguais em todos os tratamentos nas duas profundidades avaliadas (Tabela 3). Além de não ter sido constatada influência da aplicação de lodo de esgoto sobre os teores de $\mathrm{Cr}$, o aporte do metal no solo ficou bem abaixo da carga acumulada teórica permitida pela resolução Conama 375 (Brasil, 2006) de 154 $\mathrm{kg} \mathrm{ha}^{-1} \mathrm{de} \mathrm{Cr}$.

Observa-se, na Tabela 4, que apesar de os teores de Zn no solo terem variado de baixo nos tratamentos sem lodo de esgoto a alto, nos tratamentos em que foi aplicado o resíduo, plantas de

Tabela 4. Teores de metais pesados na folha e no pecíolo de girassol, em resposta à aplicação de fertilizante mineral e lodo de esgoto estabilizado de diferentes formas

\begin{tabular}{|c|c|c|c|c|c|c|c|}
\hline \multirow{3}{*}{ Metais } & \multicolumn{6}{|c|}{ Tratamentos } & \multirow{3}{*}{$\begin{array}{l}\text { CV } \\
\%\end{array}$} \\
\hline & TE & LS & LCO & LV & LCA & $A Q$ & \\
\hline & \multicolumn{6}{|c|}{$\mathrm{mg} \mathrm{kg}^{-1}$} & \\
\hline \multicolumn{8}{|c|}{ Folha } \\
\hline $\mathrm{Zn}$ & $45,55^{\mathrm{ns}}$ & $54,80^{\mathrm{ns}}$ & $48,80^{\mathrm{ns}}$ & $49,05^{\text {ns }}$ & $51,55^{\mathrm{ns}}$ & $44,55^{\mathrm{ns}}$ & 14,09 \\
\hline $\mathrm{Cu}$ & $20,25^{\text {ns }}$ & $28,50^{\text {ns }}$ & $18,25^{\mathrm{ns}}$ & $18,75^{\text {ns }}$ & $22,00^{\mathrm{ns}}$ & $19,75^{\text {ns }}$ & 32,65 \\
\hline $\mathrm{Pb}$ & $26,81^{\text {ns }}$ & $23,69^{\text {ns }}$ & $26,03^{\mathrm{ns}}$ & $29,16^{\mathrm{ns}}$ & $32,28^{\text {ns }}$ & $29,16^{\mathrm{ns}}$ & 26,50 \\
\hline $\mathrm{Cr}$ & $25,00^{\mathrm{ns}}$ & $25,00^{\mathrm{ns}}$ & $31,25^{\mathrm{ns}}$ & $50,00^{\mathrm{ns}}$ & $31,25^{\mathrm{ns}}$ & $31,25^{\mathrm{ns}}$ & 48,11 \\
\hline $\mathrm{Ni}$ & $11,17^{\text {ns }}$ & $12,38^{\text {ns }}$ & $12,62^{\mathrm{ns}}$ & $13,10^{\text {ns }}$ & $11,19^{\text {ns }}$ & $13,10^{\text {ns }}$ & 15,34 \\
\hline $\mathrm{Cd}$ & $1,31^{\mathrm{ns}}$ & $1,49^{\mathrm{ns}}$ & $1,49^{\mathrm{ns}}$ & $1,57^{\mathrm{ns}}$ & $1,37^{\mathrm{ns}}$ & $1,34^{\mathrm{ns}}$ & 27,42 \\
\hline \multicolumn{8}{|c|}{ Pecíolo } \\
\hline $\mathrm{Zn}$ & $12,80^{\text {ns }}$ & $13,80^{\text {ns }}$ & $15,55^{\mathrm{ns}}$ & $17,80^{\text {ns }}$ & $11,05^{\text {ns }}$ & $8,55^{\mathrm{ns}}$ & 35,74 \\
\hline $\mathrm{Cu}$ & $6,75 \mathrm{a}$ & $6,00 \mathrm{~b}$ & $5,25 b$ & $4,75 \mathrm{~b}$ & $7,50 \mathrm{a}$ & $5,75 b$ & 12,42 \\
\hline $\mathrm{Pb}$ & $22,66^{\text {ns }}$ & 29,37 ns & $27,94^{\mathrm{ns}}$ & $26,64^{\text {ns }}$ & $26,32^{\text {ns }}$ & $23,88^{\text {ns }}$ & 17,78 \\
\hline $\mathrm{Cr}$ & $81,20^{\text {ns }}$ & $75,00^{\text {ns }}$ & $62,50^{\mathrm{ns}}$ & $75,00^{\text {ns }}$ & $75,00^{\text {ns }}$ & $100,00^{\mathrm{ns}}$ & 33,35 \\
\hline $\mathrm{Ni}$ & $175,58^{\text {ns }}$ & $172,87^{\text {ns }}$ & $90,91^{\mathrm{ns}}$ & $133,53^{\text {ns }}$ & $148,10^{\text {ns }}$ & $164,49^{\text {ns }}$ & 35,50 \\
\hline $\mathrm{Cd}$ & $0,74^{\text {ns }}$ & $0,74^{\mathrm{ns}}$ & $0,68^{\text {ns }}$ & $0,71^{\text {ns }}$ & $0,74^{\text {ns }}$ & $0,84^{\text {ns }}$ & 30,13 \\
\hline
\end{tabular}

TE - Testemunha; LS - Lodo de esgoto solarizado; LCO - Lodo de esgoto compostado; LV - Lodo de esgoto vermicompostado; LCA - Lodo de esgoto caleado; AQ - Adubação química. ns - Não significativo Para cada nutriente, médias seguidas de mesma letra, na linha, não diferem estatisticamente entre si, a 0,05 de probabilidade pelo teste Scott-Knott 
girassol adubadas com lodo de esgoto apresentaram os mesmo teores de $\mathrm{Zn}$ nas folhas e nos pecíolos das plantas cultivadas sem adubação (testemunha) ou com adubação química. Os teores observados se mantinham dentro da faixa de 30 a $80 \mathrm{mg} \mathrm{kg}^{-1}$, considerados nutricionalmente adequados (Oliveira, 2004). Destaca-se que, mesmo no tratamento lodo de esgoto caleado cuja quantidade de $\mathrm{Zn}$ aplicada foi 3,8 vezes a recomendada, não houve aumentos de absorção do elemento pela cultura fato passível de estar relacionado à forma do elemento presente no solo uma vez que durante o processo de caleação são adicionadas grandes quantidades de cal $(\mathrm{CaOH})$ ao lodo que, quando aplicado ao solo, promove a elevação do seu $\mathrm{pH}$ favorecendo a precipitação do elemento. Murtaza et al. (2011) observaram correlação negativa entre o $\mathrm{pH}$ do solo adubado com lodo de esgoto e a disponibilidade de $\mathrm{Zn}$ promovida principalmente pelas reações de precipitação/adsorção do elemento em razão da elevação do $\mathrm{pH}$.

A aplicação de lodo de esgoto ao solo não promoveu aumento nos teores foliares de $\mathrm{Cu}$ (Tabela 4) e, com exceção do tratamento com lodo de esgoto solarizado, os níveis ficaram aquém do considerado adequado (25-100 $\mathrm{mg} \mathrm{kg}^{-1}$ ), de acordo com Oliveira (2004), apesar de o aporte de $\mathrm{Cu}$ no solo nos tratamentos com lodo de esgoto compostado e caleado ter sido aproximadamente 3 vezes superior ao recomendado para a cultura, na condição de solo do experimento. Como já discutido, o lodo de esgoto caleado promove aumento no $\mathrm{pH}$ do solo e, de acordo com estudos realizados por Borges \& Coutinho (2004a), a elevação do $\mathrm{pH}$ em solos adubados com lodo de esgoto provoca a passagem do $\mathrm{Cu}$ de formas mais fitodisponíveis (fase orgânica) para formas mais estáveis (ligado aos óxidos de $\mathrm{Fe} \mathrm{e} \mathrm{Al}$ ), sendo que esses autores constataram que somente a fração orgânica contribuiu significativamente para os teores de Cu disponíveis no solo quando da aplicação de lodo de esgoto. E mesmo quando ligado à matéria orgânica, o $\mathrm{Cu}$ tende a formar composto estável diminuindo a absorção pelas plantas. Por outro lado, os teores de $\mathrm{Cu}$ no pecíolo do girassol foram superiores quando aplicado lodo de esgoto caleado em relação às demais formas de estabilização do lodo e ao tratamento químico, embora a correlação negativa entre o pH de solo adubado com lodo de esgoto e a disponibilidade de $\mathrm{Cu}$ tenham sido observadas por Murtaza et al. (2011).

Plantas de girassol adubadas com lodo de esgoto apresentaram teores de $\mathrm{Pb}$ e $\mathrm{Cr}$ na folha e no pecíolo semelhantes às plantas do tratamento testemunha (sem adubação) e às plantas que receberam adubação química (Tabela 4), apesar de o teor de $\mathrm{Pb}$ no solo apresentar-se superior com a aplicação de lodo de esgoto caleado e solarizado. Borges \& Coutinho (2004a) constataram aumentos nos teores de $\mathrm{Pb}$ no solo em razão da aplicação de lodo de esgoto; entretanto, a elevação do $\mathrm{pH}$ proporcionou redução significativa nos teores do elemento na fração trocável e aumento na fração orgânica e óxidos, o que pode ter ocorrido no presente experimento com a aplicação de lodo de esgoto caleado resultando em diminuição na disponibilidade do elemento para as plantas de girassol, neste tratamento.

Embora não tenham sido detectados no solo, os elementos $\mathrm{Ni}$ e Cd foram detectados na folha e no pecíolo do girassol; apesar disto, os teores observados nos tratamentos com lodo de esgoto foram semelhantes aos constatados nos tratamentos químicos e testemunha (Tabela 4). Ressalta-se ainda que os teores de $\mathrm{Cr}$ e Ni no pecíolo foram, respectivamente, 2,4 e 10,0 vezes superiores aos teores constatados no limbo foliar do girassol.

\section{CONCLUSões}

1. A aplicação de lodo de esgoto solarizado, compostado, vermicompostado e caleado em quantidade suficiente para fornecer o $\mathrm{N}$ requerido pelo girassol, aumenta os teores de $\mathrm{Zn}$ no solo.

2. A aplicação de lodo de esgoto solarizado e caleado em quantidade suficiente para fornecer o $\mathrm{N}$ requerido pelo girassol, aumenta os teores de $\mathrm{Pb}$ no solo.

3. A aplicação de lodo de esgoto solarizado, compostado, vermicompostado e caleado em quantidade suficiente para fornecer o $\mathrm{N}$ requerido pelo girassol, não influencia, em geral, os teores de $\mathrm{Cu}, \mathrm{Cr}, \mathrm{Cd}, \mathrm{Ni}$, $\mathrm{Pb}$ e $\mathrm{Zn}$ na planta.

\section{Agradecimentos}

Os autores expressam seus maiores agradecimentos à FAPEMIG, ao CNPq e à CAPES, pelo apoio financeiro que possibilitou a realização deste trabalho.

\section{Literatura Citada}

Azizi, A. B.; Lim, M. P. M.; Noor, Z. M.; Abdullah, N. Vermiremoval of heavy metal in sewage sludge by utilising Lumbricus rubellus. Ecotoxicology and Environmental Safety, v.90, p.13-20, 2013.

Borges, M. R.; Coutinho, E. L. M. Metais pesados do solo após aplicação de biossólido: I. fracionamento. Revista Brasileira de Ciência do Solo, v.28, p.543-555, 2004a.

Borges, M. R.; Coutinho, E. L. M. Metais pesados do solo após aplicação de biossólido. II - Disponibilidade. Revista Brasileira de Ciência do Solo, v.28, p.557-568, 2004b.

Brasil. Ministério do Meio Ambiente. Conselho Nacional de Meio Ambiente. Resolução n. 375, de 29 de agosto de 2006. Define critérios e procedimentos, para o uso agrícola de lodos de esgoto gerados em estações de tratamento de esgoto sanitário e seus produtos derivados. Brasília, 2006. <http:// www.fundagresorg.br/biossolido/images/downloads/ res_conama37506>. 10 Nov. 2011.

Campos, F. S.; Alves, M. C.; Souza, Z. M.; Pereira, G. T. Atributos físico-hídricos de um Latossolo após a aplicação de lodo de esgoto em área degradada do Cerrado. Ciência Rural, v.41, p.796-803, 2011.

CFSEMG - Comissão de Fertilidade do Solo do Estado de Minas Gerais. Recomendação para o uso de corretivos e fertilizantes em Minas Gerais. $5^{\text {a }}$ aproximação. Viçosa, MG: CFSMG, 1999. 359p. 
Corrêa, J. C.; Bull, L. T.; Crusciol, C. A. C.; Moraes, M. H. Alteração de atributos físicos em latossolo com aplicação superficial de escória de aciaria, lama cal, lodos de esgoto e calcário. Revista Brasileira de Ciência do Solo, v.33, p.263272, 2009.

Cutright, T.; Gunda, N.; Kurt, F. Simultaneous hyper accumulation of multiple heavy metals by helianthus annuus grown in a contaminated sandy-loam soil. International Journal of Phytoremediation, v.12, p.562-573, 2010.

During, R. A.; Gath, S. Utilization of municipal organic wastes in agriculture: Where do we stand, where will we go? Journal of Plant Nutrition and Soil Science, v.165, p.544-556, 2002.

EMBRAPA - Empresa Brasileira de Pesquisa Agropecuária. Manual de métodos de análise de solo. 2.ed. Rio de Janeiro: EMBRAPA/Embrapa CNPS, 1997. 212p.

Fassler, E.; Robinson, B. H.; Stauffer, W.; Gupta, S. K.; Papritz, A.; Schulin, R. Phytomanagement of metal-contaminated agricultural land using sunflower, maize and tobacco. Agriculture, Ecosystems and Environment, v.136, p.49-58, 2010.

Fia, R.; Matos, A. T.; Aguirre, C. I. Características químicas de solo adubado com doses crescentes de lodo de esgoto caleado. Engenharia na Agricultura, v.13, p.287-299, 2005.

Galdos, M. V.; Maria, I. C.; Camargo, O. A. Atributos químicos e produção de milho em um latossolo vermelho eutroférrico tratado com lodo de esgoto. Revista Brasileira de Ciência do Solo, v.28, p.569-577, 2004.

Galrão, E. Z. Micronutrientes. In: Souza, D. M. G.; Lobado, E. (Ed.). Cerrado: Correção do solo e adubação. 2. ed. Brasília: EMBRAPA Informação Tecnológica, 2004. cap.8, p.185-226.

Gupta, R.; Garg, V. K. Stabilization of primary sewage sludge during vermicomposting. Journal of Hazardous Materials, v.153, p.1023-1030, 2008.

Haynes, R. J.; Murtaza, G.; Naidu, R. Inorganic and organic constituents and contaminants of biosolids: Implications for Land Application. Advances in Agronomy, v.104, p.165237, 2009.

Houhou, J.; Lartiges, B.S.; Montarges-Pelletier, E.; Sieliechi, J.; Ghanbaja, J.; Kohler, A. Sources, nature, and fate of heavy metal-bearing particles in the sewer system. Science of the Total Environment, v.407, p.6052-6062, 2009.

Khwairakpam, M.; Bhargava, R. Vermitechnology for sewage sludge recycling. Journal of Hazardous Materials, v.161, p.948-954, 2009.

Malavolta, E.; Vitti, G. C.; Oliveira, S. A. Avaliação do estado nutricional das plantas: Princípios e aplicações. 2. ed. Piracicaba: Potafos, 1997. 319p.
Maria, I. C.; Chiba, M. K.; Costa, A.; Berton, R. S. Sewage sludge application to agricultural land as soil physical conditioner. Revista Brasileira de Ciência do Solo, v.34, p.967-974, 2010.

Morgan, J. E.; Morgan, A. J. The distribution and intracellular compartmentation of metals in the endogeic earthworm Aporrectodea caliginosa sampled from an unpolluted and a metal-contaminated site. Environmental Pollution, v.99, p.167-175, 1998.

Murtaza, G.; Haynes, R. J.; Naidu, R.; Belyaeva, O. N.; Kim, K. R.; Lamb, D. T.; Bolan, N. S. Natural attenuation of $\mathrm{Zn}, \mathrm{Cu}$, $\mathrm{Pb}$ and $\mathrm{Cd}$ in three biosolids-amended soils of contrasting pH measured using rhizon pore water samplers. Water, Air, Soil and Pollution, v.221, p.351-363, 2011.

Nogueira, T. A. R.; Franco, A.; He, Z.; Braga, V. S.; Firme, L. P.; Abreu-Junior, C. H. short-term usage of sewage sludge as organic fertilizer to sugarcane in a tropical soil bears little threat of heavy metal contamination. Journal of Environmental Management, v.114, p.168-177, 2013.

Nogueira, T. A. R.; Sampaio, R. A.; Fonseca, I. M.; Ferreira, C. S.; Santos, S. E.; Ferreira, L. C.; Gomes, E.; Fernandes, L. A. Metais pesados e patógenos em milho e feijão caupi consorciados, adubados com lodo de esgoto. Revista Brasileira de Engenharia Agrícola e Ambiental, v.11, p.331338, 2007.

Oliveira, S. A. Análise foliar. In: Souza, D. M. G.; Lobado, E. (ed.). Cerrado: correção do solo e adubação. 2. ed. Brasília: EMBRAPA Informação Tecnológica, 2004. cap. 10, p. 245255.

Souza, Z. M.; Beutler, A. N.; Melo, V. P.; Melo, W. J. Estabilidade de agregados e resistência à penetração em Latossolos adubados por cinco anos com biossólido. Revista Brasileira de Ciência do Solo, v.29, p.117-123, 2005.

Tedesco, M. J.; Gianello, C.; Bissani, C. A.; Bohnen, H.; Volkweiss, S. J. Análise de solo, plantas e outros materiais. 2. ed. Porto Alegre: Departamento de Solos/UFRGS, 1995. 174p. Boletim Técnico, 5

USEPA - United States Environmental Protection Agency. Title 40 CFR: part 503: final rules standards for the use for disposal of sewage sludge. Washington, 1999. <http://www. epa.gov/>. 10 Set. 2011.

Vijver, M. G.; Vink, J. P. M.; Miermans, C. J. H.; Gestel, C. A. M. van. Metal accumulation in earthworms inhabiting floodplain soils. Environmental Pollution, v.148, p.132-140, 2007.

Zuba Junio, G. R.; Sampaio, R. A.; Santos, G. B.; Nascimento, A. L.; Prates, F. B. S.; Fernandes, L. A. Metais pesados em milho fertilizado com fosfato natural e composto de lodo de esgoto. Revista Brasileira de Engenharia Agrícola e Ambiental, v.15, p.1082-1088, 2011. 